\title{
New Versatile Pd-Catalyzed Alkylation of Indoles via Nucleophilic Allylic Substitution. In Controlling the Regioselectivity
}

\author{
Marco Bandini,* Alfonso Melloni, Achille Umani-Ronchi* \\ Dipartimento di Chimica “G. Ciamician”, via Selmi 2 - 40126, Bologna, Italy \\ marco.bandini@unibo.it; achille.umanironchi@unibo.it
}

\section{Supporting Information}

\section{General.}

$\mathrm{CH}_{2} \mathrm{Cl}_{2}$, toluene, THF, $\mathrm{Et}_{2} \mathrm{O}$ and DMF were supplied by Fluka in Sureseal ${ }^{\circledR}$ bottles and used as received. All starting materials and reagents were obtained from Aldrich and Fluka and used without further purification. All the allylic carbonates were prepared following a procedure reported in the literature. ${ }^{1}{ }^{1} \mathrm{H}-\mathrm{NMR}$ spectra were recorded on Varian $200(200 \mathrm{MHz})$ or Varian 300 (300 $\mathrm{MHz}$ ) spectrometers. Chemical shifts are reported in ppm from TMS with the solvent resonance as the internal standard (deuterochloroform: $\delta 7.27 \mathrm{ppm}$ ). Data are reported as follows: chemical shift, multiplicity $(\mathrm{s}=$ singlet, $\mathrm{d}=$ doublet, $\mathrm{t}=$ triplet, $\mathrm{q}=$ quartet, $\mathrm{br}=$ broad, $\mathrm{m}=$ multiplet $)$, coupling constants $(\mathrm{Hz}) .{ }^{13} \mathrm{C}-\mathrm{NMR}$ spectra were recorded on a Varian $200(50 \mathrm{MHz})$ or Varian $300(75$ $\mathrm{MHz}$ ) spectrometers with complete proton decoupling. Chemical shifts are reported in ppm from TMS with the solvent as the internal standard (deuterochloroform: $\delta 77.0 \mathrm{ppm}$ ). GC-mass spectra were performed at an ionizing voltage of $70 \mathrm{eV}$. LC-electrospray ionization mass spectra were obtained with Agilent Technologies MSD1100 single-quadrupole mass spectrometer. Chromatographic purification was done with 70-230 mesh aluminiun oxide 90 mesh allumina or with 240-400 mesh silica gel. IR analysis were performed with a FT-IR spectrophotometer. IR spectra are expressed by wavenumber $\left(\mathrm{cm}^{-1}\right)$. Elemental analyses were carried out by using a EACE 1110 CHNOS analyzer. Melting points are uncorrected. 


\section{General procedure for the $C-3$ alkylation of indoles by 1,3-diphenylprop-2-enyl methyl}

carbonate (2a).

All the reactions were carried out at reflux under nitrogen atmosphere. A solution of $[\mathrm{PdCl}(\pi-$ allyl) $]_{2}\left(7.5 \cdot 10^{-3} \mathrm{mmol}, 0.05\right.$ equiv) and 1,2-bis(diphenylphosphino)-ethane (dppe) $\left(1.65 \cdot 10^{-2} \mathrm{mmol}\right.$, 0.11 equiv) in the solvent of choice (anhydrous THF or $\mathrm{CH}_{2} \mathrm{Cl}_{2}, 1.0 \mathrm{~mL}$ ) was stirred for $30 \mathrm{~min}$ and then $2 \mathbf{a}(0.3 \mathrm{mmol}, 2.0$ equiv) was added. The yellow solution was stirred for ca $30 \mathrm{~min}$, followed by the addition of the desired indole $\mathbf{1 a - h}\left(0.15 \mathrm{mmol}, 1.0\right.$ equiv) and $\mathrm{Li}_{2} \mathrm{CO}_{3}(0.3 \mathrm{mmol}, 2.0$ equiv). The resulting reaction mixture was refluxed for $24 \mathrm{~h}$ and then quenched with water $(4 \mathrm{~mL})$ and extracted with AcOEt. The combined organics were dried over $\mathrm{Na}_{2} \mathrm{SO}_{4}$, concentrated under reduced pressure and purified by flash chromatography on alumina.

\section{3-((E)-1,3-Diphenyl-allyl)-1H-indole (3a)}

Colorless oil. $\mathrm{R}_{\mathrm{f}} 0.3$ (n-Hexane:AcOEt 90:10).

${ }^{1} \mathrm{H}-\mathrm{NMR}\left(300 \mathrm{MHz}, \mathrm{CDCl}_{3}\right): \delta 5.05(\mathrm{~d}, 1 \mathrm{H}, J=7.5 \mathrm{~Hz}), 6.34-6.40(\mathrm{~m}, 1 \mathrm{H}), 6.66(\mathrm{dd}, 1 \mathrm{H}, J=15.9$, $7.2 \mathrm{~Hz}), 6.80-7.01(\mathrm{~m}, 3 \mathrm{H}), 7.03-7.26(\mathrm{~m}, 12 \mathrm{H}), 7.91$ (br s, $1 \mathrm{H}) .{ }^{13} \mathrm{C}-\mathrm{NMR}\left(50 \mathrm{MHz}, \mathrm{CDCl}_{3}\right): \delta$ 46.1, 111.1, 118.5, 119.3, 119.8, 122.0, 122.6, 126.3 (2 C), 126.6, 126.7, 127.2, 127.7, 128.4, 130.5, 132.5, 136.6, 137.4, 143.3. IR (neat): v 3429, 3067, 2903, 2863, 2727, 1685, 1618, 1499, 1208, 1128, 971, $740 \mathrm{~cm}^{-1}$. Anal. calcd for $\left(\mathrm{C}_{23} \mathrm{H}_{19} \mathrm{~N}\right)$ : C, 89.28; H, 6.19; N, 4.53; Found: C, 89.22; H, $6.13 ; \mathrm{N}, 4.51$.

\section{3-((E)-1,3-Diphenyl-allyl)-2-methyl-1H-indole (3b)}

Pale yellow oil. $\mathrm{R}_{\mathrm{f}} 0.3$ (n-Hexane:AcOEt 95:5).

${ }^{1} \mathrm{H}-\mathrm{NMR}\left(200 \mathrm{MHz}, \mathrm{CDCl}_{3}\right): \delta 2.39(\mathrm{~s}, 3 \mathrm{H}), 5.14(\mathrm{~d}, 1 \mathrm{H}, J=7.0 \mathrm{~Hz}), 6.35-6.51(\mathrm{~m}, 1 \mathrm{H}), 6.78-7.05$ $(\mathrm{m}, 2 \mathrm{H}), 7.06-7.54(\mathrm{~m}, 13 \mathrm{H}), 7.81$ (br s, $1 \mathrm{H}) .{ }^{13} \mathrm{C}-\mathrm{NMR}\left(50 \mathrm{MHz}, \mathrm{CDCl}_{3}\right): \delta 12.4,45.0,110.2$, $112.9,119.2$, 119.4, 120.9, 126.0, 126.3, 127.0, 128.3, 128.4, 128.5, 128.6, 130.6, 131.6, 132.1, 135.3, 137.5, 143.4. IR (neat): $v$ 3409, 3025, 2923, 2854, 1695, 1618, 1459, 1264, $968 \mathrm{~cm}^{-1}$. Anal. calcd for $\left(\mathrm{C}_{24} \mathrm{H}_{21} \mathrm{~N}\right)$ : C, 89.12; H, 6.54; N, 4.33; Found: C, 89.10; H, 6.48; N, 4.31.

\section{3-((E)-1,3-Diphenyl-allyl)-2-phenyl-1H-indole (3c)}

Pale yellow solid. Mp 64-66 ${ }^{\circ} \mathrm{C} .0 .3$ (n-Hexane:AcOEt 95:5).

${ }^{1} \mathrm{H}-\mathrm{NMR}\left(200 \mathrm{MHz}, \mathrm{CDCl}_{3}\right): \delta 5.31(\mathrm{~d}, 1 \mathrm{H}, J=7.0 \mathrm{~Hz}), 6.38-6.47(\mathrm{~m}, 1 \mathrm{H}), 6.85-7.06(\mathrm{~m}, 2 \mathrm{H})$, 7.15-7.60 (m, 18H), $8.12(\mathrm{~s}, 1 \mathrm{H}) .{ }^{13} \mathrm{C}-\mathrm{NMR}\left(50 \mathrm{MHz}, \mathrm{CDCl}_{3}\right): \delta 45.1,110.9,113.9,119.7,121.2$, $122.1,126.1,126.3,127.1,127.9,128.0,128.2$, 128.3, 128.4, 128.6, 128.8, 131.1, 132.3, 132.9, 
135.6, 136.3, 137.5, 143.5. IR (nujol): v 3421, 3050, 2923, 2853, 1600, 1490, 1457, 1307, 1023, $966 \mathrm{~cm}^{-1}$. Anal. calcd for $\left(\mathrm{C}_{29} \mathrm{H}_{23} \mathrm{~N}\right)$ : C, 90.35; H, 6.01; N, 3.63; Found: C, 90.28; H, 5.97; N, 3.62.

\section{5-Benzyloxy-3-((E)-1,3-diphenyl-allyl)-1H-indole (3d)}

Colorless oil. $\mathrm{R}_{\mathrm{f}} 0.3$ (n-Hexane:AcOEt 95:5).

${ }^{1} \mathrm{H}-\mathrm{NMR}\left(200 \mathrm{MHz}, \mathrm{CDCl}_{3}\right): \delta 4.94(\mathrm{~s}, 2 \mathrm{H}), 5.04(\mathrm{~d}, 1 \mathrm{H}, J=7.2 \mathrm{~Hz}), 6.31-6.48(\mathrm{~m}, 1 \mathrm{H}), 6.70(\mathrm{dd}$, $1 \mathrm{H}, J=15.6,6.8 \mathrm{~Hz}), 6.78-6.98(\mathrm{~m}, 3 \mathrm{H}), 7.06-7.50$ (m, 16H), 7.86 (br s, $1 \mathrm{H}) .{ }^{13} \mathrm{C}-\mathrm{NMR}(50 \mathrm{MHz}$, $\left.\mathrm{CDCl}_{3}\right): \delta 46.2,70.8,103.4,111.7,112.9,118.3,123.4,126.3(2 \mathrm{C}), 126.6,127.1,127.6(2 \mathrm{C}$ ), 127.7, 128.4, 128.5, 130.5, 131.5, 132.0, 132.4, 137.5, 137.6, 143.3, 152.9. IR (neat): $v$ 3427, 3058, 3026, 2925, 2859, 1598, 1487, 1481, 1379, 1264, 1183, 1026, $968 \mathrm{~cm}^{-1}$. Anal. calcd for $\left(\mathrm{C}_{30} \mathrm{H}_{25} \mathrm{NO}\right): \mathrm{C}, 86.71 ; \mathrm{H}, 6.06 ; \mathrm{N}, 3.37$; Found: $\mathrm{C}, 86.68 ; \mathrm{H}, 6.02 ; \mathrm{N}, 3.35$.

\section{5-Bromo-3-((E)-1,3-diphenyl-allyl)-1H-indole (3e)}

Colorless oil. $\mathrm{R}_{\mathrm{f}} 0.3$ (n-Hexane:AcOEt 85:15).

${ }^{1} \mathrm{H}-\mathrm{NMR}\left(200 \mathrm{MHz}, \mathrm{CDCl}_{3}\right): \delta 5.02(\mathrm{~d}, 1 \mathrm{H}, J=7.0 \mathrm{~Hz}), 6.28-6.50(\mathrm{~m}, 1 \mathrm{H}), 6.64(\mathrm{dd}, 1 \mathrm{H}, J=15.8$, $7.4 \mathrm{~Hz}), 6.87$ (m, 1H), 7.08-7.55 (m, 13H), 7.98 (br s, 1H). ${ }^{13} \mathrm{C}-\mathrm{NMR}\left(50 \mathrm{MHz}, \mathrm{CDCl}_{3}\right): \delta 45.8$, $112.5,112.7,118.5,122.3,123.8,125.0,126.3$ (2 C), 126.5, 127.2, 128.4, 128.5 (2 C), 130.8, 132.0, 135.2, 137.3, 142.9. IR (nujol): $v$ 3414, 3018, 2859, 1513, 1471, 1268, 1221, 1093, $971 \mathrm{~cm}^{-1}$. Anal. calcd for $\left(\mathrm{C}_{23} \mathrm{H}_{18} \mathrm{BrN}\right)$ : C, 71.14; H, 4.67; N, 3.61; Found: C, 71.10; H, 4.64; N, 3.58.

\section{5-Cyano-3-((E)-1,3-diphenyl-allyl)-1H-indole (3f)}

Colorless oil. $\mathrm{R}_{\mathrm{f}} 0.3$ ( $n$-Hexane:AcOEt 85:15).

${ }^{1} \mathrm{H}-\mathrm{NMR}\left(200 \mathrm{MHz}, \mathrm{CDCl}_{3}\right): \delta 5.05(\mathrm{~d}, 1 \mathrm{H}, J=7.0 \mathrm{~Hz}), 6.34-6.42(\mathrm{~m}, 1 \mathrm{H}), 6.64(\mathrm{dd}, 1 \mathrm{H}, J=15.8$, $7.2 \mathrm{~Hz}), 7.02(\mathrm{~m}, 1 \mathrm{H}), 7.16-7.41(\mathrm{~m}, 12 \mathrm{H}), 7.69(\mathrm{~m}, 1 \mathrm{H}), 8.39$ (br s, 1H). ${ }^{13} \mathrm{C}-\mathrm{NMR}(50 \mathrm{MHz}$, $\left.\mathrm{CDCl}_{3}\right): \delta 45.9,102.5,112.0,119.8,120.7,124.7,125.1,125.5,126.3,126.6,126.8,127.4,128.3$, 128.5, 128.6, 131.2, 131.5, 137.1, 138.4, 142.5. IR (neat): v 3408, 3332, 3058, 3026, 2932, 2853, 2220, 1706, 1616, 1470, 1361, 1222, 1094, $909 \mathrm{~cm}^{-1}$. Anal. calcd for $\left(\mathrm{C}_{24} \mathrm{H}_{18} \mathrm{~N}_{2}\right): \mathrm{C}, 86.20 ; \mathrm{H}, 5.43$; N, 8.38; Found: C, 86.14; H, 5.38; N, 8.37.

\section{3-((E)-1,3-Diphenyl-allyl)-1-methyl-1H-indole (3g)}

Pale yellow oil. $\mathrm{R}_{\mathrm{f}} 0.3$ ( $n$-Hexane:AcOEt 97:3).

${ }^{1} \mathrm{H}-\mathrm{NMR}\left(300 \mathrm{MHz}, \mathrm{CDCl}_{3}\right): \delta 3.77(\mathrm{~s}, 3 \mathrm{H}), 5.15(\mathrm{~d}, 1 \mathrm{H}, J=7.2 \mathrm{~Hz}), 6.45-6.51(\mathrm{~m}, 1 \mathrm{H}), 6.73-6.81$ (m, 2H), 7.03-7.24 (m, 1H), 7.17-7.58 (m, 13H). ${ }^{13} \mathrm{C}-\mathrm{NMR}\left(75 \mathrm{MHz}, \mathrm{CDCl}_{3}\right): \delta 46.1,56.4,109.1$, 118.8 , 121.6, 126.2, 126.3, 126.5, 126.8, 127.1, 127.2, 127.3, 128.4, 128.5, 130.1, 130.4, 131.4, 
132.7, 137.5, 143.5. IR (neat): v 3418, 3051, 2920, 1741, 1610, 1437, 1351, 1094, $980 \mathrm{~cm}^{-1}$. Anal. calcd for $\left(\mathrm{C}_{24} \mathrm{H}_{21} \mathrm{~N}\right)$ : C, 89.12; H, 6.54; N, 4.33; Found: C, 89.05; H, 6.48; N, 4.35.

\section{Preparation of 3-((E)-1-methyl-but-2-enyl)-1H-indole (4)}

The reaction was carried out under nitrogen atmosphere. A solution of $[\mathrm{PdCl}(\pi \text {-allyl })]_{2}\left(7.5 \cdot 10^{-3}\right.$ mmol, 0.05 equiv) and triphenyl phosphine $\left(3.30 \cdot 10^{-2} \mathrm{mmol}, 0.22\right.$ equiv) in anhydrous $\mathrm{CH}_{2} \mathrm{Cl}_{2}(0.5$ $\mathrm{mL})$ was stirred for $30 \mathrm{~min}$ and then $\mathbf{2 c}(0.3 \mathrm{mmol}, 2.0$ equiv) was added. The yellow solution was stirred for ca $30 \mathrm{~min}$, followed by the addition of $\mathbf{1 a}\left(0.15 \mathrm{mmol}, 1.0\right.$ equiv), $\mathrm{Li}_{2} \mathrm{CO}_{3}$ (0.3 mmol, 2.0 equiv) and BSA ( $0.3 \mathrm{mmol}, 2.0$ equiv). The resulting reaction mixture was stirred for $8 \mathrm{~h}$ at reflux (complete consumption of the indole as judged by TLC) and then quenched with water. Usual work-up and purification by flash chromatography (silica gel, $n$-Hexane:AcOEt 95:5) afforded 4 as a light brown oil in $80 \%$ yield, $22 \mathrm{mg}$ (4:5 ratio $16: 1)$.

${ }^{1} \mathrm{H}-\mathrm{NMR}\left(200 \mathrm{MHz}, \mathrm{CDCl}_{3}\right): \delta 1.34(\mathrm{~d}, 3 \mathrm{H}, J=7.0 \mathrm{~Hz}), 1.6(\mathrm{~d}, 3 \mathrm{H}, J=5.6 \mathrm{~Hz}), 3.64(\mathrm{~m}, 1 \mathrm{H})$, 5.37-5.69 (m, 2H), $6.85(\mathrm{~m}, 1 \mathrm{H}), 6.98-7.31(\mathrm{~m}, 3 \mathrm{H}), 7.58(\mathrm{~m}, 1 \mathrm{H}), 7.81(\mathrm{br} \mathrm{s}, 1 \mathrm{H}) .{ }^{13} \mathrm{C}-\mathrm{NMR}(50$ $\left.\mathrm{MHz}, \mathrm{CDCl}_{3}\right): \delta 17.8,20.9,33.9,111.1,119.0,119.6,120.1,121.2,121.8,123.2,126.8,136.2$, 136.5. IR (neat): $v$ 3416, 3058, 3018, 2963, 2932, 2872, 1618, 1456, 1414, 1337, 121, 1094, 1009, $966 \mathrm{~cm}^{-1}$. EI-MS (relative intensity): 115 (26), 144 (39), 155 (33), 170 (100), 185 (55, M). Anal. calcd for $\left(\mathrm{C}_{13} \mathrm{H}_{15} \mathrm{~N}\right)$ : C, 84.28; H, 8.16; N, 7.56; Found: C, 84.21; H, 8.09; N, 7.55.

\section{Preparation of 1-((E)-1-methyl-but-2-enyl)-1H-indole (5)}

The reaction was carried out under nitrogen atmosphere. A solution of $[\mathrm{PdCl}(\pi \text {-allyl })]_{2}\left(7.5 \cdot 10^{-3}\right.$ mmol, 0.05 equiv) and dppe $\left(1.65 \cdot 10^{-2} \mathrm{mmol}, 0.11\right.$ equiv) in anhydrous THF $(1.0 \mathrm{~mL})$ was stirred for $30 \mathrm{~min}$ and then $\mathbf{2 c}(0.3 \mathrm{mmol}, 2.0$ equiv) was added. The bright yellow solution was stirred for ca $30 \mathrm{~min}$, followed by the addition of $\mathbf{1 a}\left(0.15 \mathrm{mmol}, 1.0\right.$ equiv) and $\mathrm{K}_{2} \mathrm{CO}_{3}(0.3 \mathrm{mmol}, 2.0$ equiv). The resulting reaction mixture was stirred for $12 \mathrm{~h}$ at reflux (complete consumption of the indole as judged by TLC) and then quenched with water. Usual work-up and purification by flash chromatography (silica gel, $n$-Hexane:AcOEt 99:1) afforded $\mathbf{5}$ as a colorless oil in 90\% yield, 25 $\operatorname{mg}(4: 5$ ratio $>1: 50)$.

${ }^{1} \mathrm{H}-\mathrm{NMR}\left(300 \mathrm{MHz}, \mathrm{CDCl}_{3}\right): \delta 1.53(\mathrm{~d}, 3 \mathrm{H}, J=6.3 \mathrm{~Hz}), 1.61(\mathrm{~d}, 3 \mathrm{H}, J=6.0 \mathrm{~Hz}), 4.96(\mathrm{~m}, 1 \mathrm{H})$, 5.40-5.64 (m, 2H), $6.44(\mathrm{~m}, 1 \mathrm{H}), 6.98-7.18(\mathrm{~m}, 3 \mathrm{H}), 7.28-7.31(\mathrm{~m}, 1 \mathrm{H}), 7.54-7.58(\mathrm{~m}, 1 \mathrm{H}) .{ }^{13} \mathrm{C}-$ NMR $\left(50 \mathrm{MHz}, \mathrm{CDCl}_{3}\right): \delta 16.7,20.0,48.4,101.5,109.6,119.4,121.0,121.6,126.5,127.7,128.4$, 132.3, 136.2. IR (neat): v 3063, 3020, 2941, 2830, 1654, 1654, 1601, 1528, 1449, 1404, 1221, 1212, 
1048, $893 \mathrm{~cm}^{-1}$ EI-MS (relative intensity): 69 (24), 117 (100), 185 (24, M). Anal. calcd for $\left(\mathrm{C}_{13} \mathrm{H}_{15} \mathrm{~N}\right)$ : C, 84.28; H, 8.16; N, 7.56; Found: C, 84.25; H, 8.11; N, 7.58.

\section{Preparation of 3-cyclohex-2-enyl-1H-indole (6)}

Prepared by following the alkylation procedure described above for $\mathbf{4}$, with addition of allylic carbonate $\mathbf{2 d}$ instead of $\mathbf{2 c}$. The reaction was quenched after $24 \mathrm{~h}$ at reflux (complete consumption of the indole as judged by TLC). Usual work-up and purification by flash chromatography (silica gel, n-Hexane:AcOEt 95:5) afforded 6 as a light brown oil in 89\% yield, $26 \mathrm{mg}$ (6:7 ratio 8:1).

${ }^{1} \mathrm{H}-\mathrm{NMR}\left(300 \mathrm{MHz}, \mathrm{CDCl}_{3}\right): \delta 1.49-1.80(\mathrm{~m}, 3 \mathrm{H}), 1.90-2.06(\mathrm{~m}, 3 \mathrm{H}), 3.61-3.73(\mathrm{~m}, 1 \mathrm{H}), 5.79(\mathrm{~m}$, 2H), $6.88(\mathrm{~m}, 1 \mathrm{H}), 6.98-7.38(\mathrm{~m}, 3 \mathrm{H}), 7.54-7.86(\mathrm{~m}, 1 \mathrm{H}), 7.85$ (br s, $1 \mathrm{H}) .{ }^{13} \mathrm{C}-\mathrm{NMR}(50 \mathrm{MHz}$, $\left.\mathrm{CDCl}_{3}\right): \delta 20.8,25.2,30.1,32.6,111.1,119.0,119.2,120.9,121.3,121.8,126.6,127.5,130.3$, 136.6. IR (neat): v 3420, 3038, 3008, 2960, 2922, 2865, 1616, 1444, 1408, 1331, 1208, 1088, 972 $\mathrm{cm}^{-1}$. EI-MS (relative intensity): 63 (8), 83 (17), 117 (48), 168 (100), 197 (100, M). Anal. calcd for $\left(\mathrm{C}_{14} \mathrm{H}_{15} \mathrm{~N}\right)$ : C, 85.24; H, 7.66; N, 7.10; Found: C, 85.21; H, 7.61; N, 7.08.

\section{Preparation of 1-cyclohex-2-enyl-1H-indole (7)}

The reaction was carried out under nitrogen atmosphere. A solution of $[\mathrm{PdCl}(\pi \text {-allyl })]_{2}\left(7.5 \cdot 10^{-3}\right.$ mmol, 0.05 equiv) and triphenyl phosphine $\left(3.30 \cdot 10^{-2} \mathrm{mmol}, 0.22\right.$ equiv) in anhydrous DMF (1.0 $\mathrm{mL}$ ) was stirred for $30 \mathrm{~min}$ and then $\mathbf{2 d}$ ( $0.3 \mathrm{mmol}, 2.0$ equiv) was added. The bright yellow solution was stirred for ca $30 \mathrm{~min}$, followed by the addition of $1 \mathrm{a}(0.15 \mathrm{mmol}, 1.0$ equiv) and $\mathrm{Cs}_{2} \mathrm{CO}_{3}$ (0.3 mmol, 2.0 equiv). The resulting reaction mixture was stirred for $24 \mathrm{~h}$ at $\mathrm{rt}$ (complete consumption of the indole as judged by TLC) and then quenched with water. Usual work-up and purification by flash chromatography (silica gel, $n$-Hexane:AcOEt 99:1) afforded 7 as a colorless oil in $91 \%$ yield, $27 \mathrm{mg}(\mathbf{6}: 7$ ratio $1: 12)$.

${ }^{1} \mathrm{H}-\mathrm{NMR}\left(300 \mathrm{MHz}, \mathrm{CDCl}_{3}\right): \delta$ 1.62-1.85 (m, 2H), 1.86-2.05 (m, 1H), 2.07-2.32 (m, 3H), $5.08(\mathrm{~m}$, $1 \mathrm{H})$, 5.82-5.92 (m, 1H), 6.07-6.20 (m, 1H), $6.53(\mathrm{~m}, 1 \mathrm{H}), 7.10-7.30(\mathrm{~m}, 3 \mathrm{H}), 7.40-7.50(\mathrm{~m}, 1 \mathrm{H})$, 7.68-7.78 (m, 1H). ${ }^{13} \mathrm{C}-\mathrm{NMR}\left(50 \mathrm{MHz}, \mathrm{CDCl}_{3}\right): \delta 19.9,24.9,29.9,51.2,100.6,109.6,119.3,120.9$, 121.1, 126.2, 126.6, 129.0, 132.2, 135.4. IR (neat): v 3051, 3025, 2936, 2866, 2833, 1609, 1508, 1458, 1404, 1309, 1288, 1227, 1211, $882 \mathrm{~cm}^{-1}$ EI-MS (relative intensity): 81 (26), 117 (100), 197 (31, M). Anal. calcd for $\left(\mathrm{C}_{14} \mathrm{H}_{15} \mathrm{~N}\right)$ : C, 85.24; H, 7.66; N, 7.10; Found: C, 85.18; H, 7.58; N, 7.12. 


\section{Preparation of 3-((E)-1-Methyl-3-phenyl-allyl)-1H-indole (8)}

Prepared by following the alkylation procedure described above for $\mathbf{4}$, with addition of allylic carbonate $\mathbf{2 e}$ instead of $\mathbf{2 c}$. The reaction was quenched after $14 \mathrm{~h}$ at reflux (complete consumption of the indole as judged by TLC). Usual work-up and purification by flash chromatography (silica gel, $n$-Hexane:AcOEt 95:5) afforded $\mathbf{8}$ as a light brown oil in 82\% yield, $30 \mathrm{mg}$ (8:9 ratio >10:1).

Spectral data for $\mathbf{8}$ were consistent with data reported in the literature. ${ }^{2}$

\section{Preparation of 1-((E)-1-Methyl-3-phenyl-allyl)-1H-indole (9)}

Prepared by following the alkylation procedure described above for 7 , with addition of allylic carbonate $\mathbf{2 e}$ instead of $\mathbf{2 d}$. The reaction was quenched after $20 \mathrm{~h}$ at $\mathrm{rt}$ (complete consumption of the indole as judged by TLC). Usual work-up and purification by flash chromatography ( $n$ Hexane:AcOEt 99:1) afforded 9 as a light yellow oil in 84\% yield, $31 \mathrm{mg}$ (8:9 ratio 1: 50).

${ }^{1} \mathrm{H}-\mathrm{NMR}\left(200 \mathrm{MHz}, \mathrm{CDCl}_{3}\right): \delta 1.78(\mathrm{~d}, 3 \mathrm{H}, J=7.0 \mathrm{~Hz}), 5.28(\mathrm{dq}, 1 \mathrm{H}, J=7.0,3.0 \mathrm{~Hz}), 6.46(\mathrm{~m}$, $2 \mathrm{H}), 6.60(\mathrm{~m}, 1 \mathrm{H}), 7.06-7.48(\mathrm{~m}, 9 \mathrm{H}), 7.61-7.73(\mathrm{~m}, 1 \mathrm{H}) .{ }^{13} \mathrm{C}-\mathrm{NMR}\left(50 \mathrm{MHz}, \mathrm{CDCl}_{3}\right): \delta 20.3,52.8$, 101.6, 109.8, 119.4, 120.9, 121.4, 124.6, 126.5, 127.8, 128.6, 128.7, 130.3, 130.5, 135.7, 136.4. IR (neat): $v$ 3053, 3025, 2975, 2929, 1601, 1508, 1476, 1459, 1309, 1218, $965 \mathrm{~cm}^{-1}$. EI-MS (relative intensity): 91 (43), 131 (100), $247(21, \mathrm{M})$. Anal. calcd for $\left(\mathrm{C}_{18} \mathrm{H}_{17} \mathrm{~N}\right)$ : C, 87.41; H, 6.93; N, 5.66; Found: C, 87.38; H, 6.88; N, 6.90.

\section{Preparation of benzyl-(1H-indol-2-yl-methyl)-amine (11)}

To a mixture of $\mathbf{1 0}^{3}$ (6.9 mmol, 1.0 equiv), and $\mathrm{MgSO}_{4}$ (34.5 mmol, 5.0 equiv) in toluene (40 $\mathrm{mL}$ ), benzylamine (10.35 mmol, 1.5 equiv) was added. The reaction was kept under reflux for $48 \mathrm{~h}$ (complete consumption of $\mathbf{1 0}$ as judged by GC-MS). The solvent was then evaporated under reduced pressure to give a dark viscous oil $\left({ }^{1} \mathrm{H}-\mathrm{NMR}\right.$ data of the crude were consistent with data of the benzylimine reported in the literature $).{ }^{4}$ The crude was dissolved in methanol $(50 \mathrm{~mL})$ and then $\mathrm{NaBH}_{4}$ (13.8 mmol, 2.0 equiv) was added at $0^{\circ} \mathrm{C}$ portionwise. The reaction mixture was stirred at $\mathrm{rt}$ for $6 \mathrm{~h}$ and then quenched with water $(10 \mathrm{~mL})$. Methanol was removed under reduced pressure, the aqueous phase was extracted with AcOEt, the combined organics were dried over $\mathrm{Na}_{2} \mathrm{SO}_{4}$ and then concentrated to give a brown oil which was dissolved in cyclohexane. The unsoluble solid was filtered off and evaporation of the solvent gave $\mathbf{1 1}$ as a red/brown viscous oil in $89 \%$ yield, $1.45 \mathrm{~g}$ (starting from 10).

${ }^{1} \mathrm{H}-\mathrm{NMR}\left(300 \mathrm{MHz}, \mathrm{CDCl}_{3}\right.$ ): $\delta 3.83$ (s, 2H), 3.99 (s, 2H), 3.36 (s, 1H), 7.09-7.18 (m, 2H), 7.30$7.39(\mathrm{~m}, 6 \mathrm{H}), 7.58$ (d, 1H, $J=1.5 \mathrm{~Hz}), 8.59$ (br, $1 \mathrm{H}) .{ }^{13} \mathrm{C}-\mathrm{NMR}\left(75 \mathrm{MHz}, \mathrm{CDCl}_{3}\right): \delta 46.1,53.1$, 
$100.4,110.7,119.6,120.1,121.4,121.6,128.2,128.4,128.5,136.0,137.3,139.7$. IR (nujol): $v$ $3177,2930,1481,1281,1088 \mathrm{~cm}^{-1}$. Anal. calcd for $\left(\mathrm{C}_{16} \mathrm{H}_{16} \mathrm{~N}_{2}\right): \mathrm{C}, 81.32 ; \mathrm{H}, 6.82 ; \mathrm{N}, 11.85$; Found: C, 81.28; H, 6.77; N, 11.81 .

\section{Preparation of carbonic acid $(E)-4$-[benzyl-(1H-indol-2-yl-methyl)-amino]-but-2-enyl methyl ester (12)}

To a solution of 11 (2.5 mmol, 1.0 equiv) and $\mathrm{Et}_{3} \mathrm{~N}$ (3.0 mmol, 1.2 equiv) in dry THF (30 mL), ethyl 4-bromocrotonate $\left(5.0 \mathrm{mmol}, 2.0\right.$ equiv) was added at $0^{\circ} \mathrm{C}$. The ice bath was removed and the reaction mixture was stirred at $\mathrm{rt}$ for $4 \mathrm{~h}$. Finally, the mixture was filtered and solid residue washed with $\mathrm{Et}_{2} \mathrm{O}$. Evaporation of the volatiles under reduced pressure and subsequent purification by flashchromatography (silica gel, cyclohexane:AcOEt 85:15) afforded the (E)-4-[benzyl-(1H-indol-2-ylmethyl)-amino]-but-2-enoic acid ethyl ester as a brown oil in 70\% yield, $638 \mathrm{mg}$. [ ${ }^{1} \mathrm{H}-\mathrm{NMR}, 200$ MHz: $\delta 1.30(\mathrm{t}, J=6.8 \mathrm{~Hz}, 3 \mathrm{H}), 3.26(\mathrm{~d}, J=6.0 \mathrm{~Hz}, 2 \mathrm{H}), 3.65(\mathrm{~s}, 2 \mathrm{H}), 3.76(\mathrm{~s}, 2 \mathrm{H}), 4.19(\mathrm{q}, J=7.0$ $\mathrm{Hz}, 2 \mathrm{H}), 6.08$ (dd, $J=14.8,2.2 \mathrm{~Hz}, 1 \mathrm{H}), 6.40$ (s, 1H), 6.97-7.05 (m, 1H), 7.09-7.14 (m, 2H), 7.21$7.35(\mathrm{~m}, 6 \mathrm{H}), 7.56(\mathrm{~d}, J=7.4 \mathrm{~Hz}, 1 \mathrm{H}), 8.37$ (br, 1H). ${ }^{13} \mathrm{C}-\mathrm{NMR}, 50 \mathrm{MHz}: \delta 14.3,51.3,54.2,58.3$, $60.4,101.7,110.7,119.7,120.1,121.6,123.3,127.3,128.5$ (2C), 128.8 (2C), 136.0, 138.2, 145.5, 166.1].

The ethyl ester (1.7 mmol, 1.0 equiv) was dissolved in dry toluene $(20 \mathrm{~mL})$ and then DIBAL (1.0 M in hexane, $4.25 \mathrm{mmol}, 2.5$ equiv) was added dropwise at $-78^{\circ} \mathrm{C}$. The reaction was stirred at $-78^{\circ} \mathrm{C}$ for $1 \mathrm{~h}$ (complete consumption of the ester as judged by TLC) and then quenched with $0.5 \mathrm{~mL}$ of acetic acid: $\mathrm{H}_{2} \mathrm{O}$ (1:1 mixture). The organics were washed with $\mathrm{H}_{2} \mathrm{O}$ concentrated under reduced pressure and purified by flash chromatography (silica gel, $\mathrm{CH}_{2} \mathrm{Cl}_{2}: \mathrm{MeOH} 9: 1$ ) to afford (E)-4[benzyl-(1H-indol-2-ylmethyl)-amino]-but-2-en-1-ol as a viscous oil in $82 \%$ yield, $426 \mathrm{mg}$. [ ${ }^{1} \mathrm{H}$ NMR, 300 MHz: $\delta 3.13(\mathrm{~d}, J=4.8 \mathrm{~Hz}, 2 \mathrm{H}), 3.65(\mathrm{~s}, 2 \mathrm{H}), 3.76(\mathrm{~s}, 2 \mathrm{H}), 4.11(\mathrm{~d}, J=5.2 \mathrm{~Hz}, 2 \mathrm{H})$, 5.77-5.81 (m, 2H), $6.37(\mathrm{~d}, J=1.2 \mathrm{~Hz}, 1 \mathrm{H}), 7.08-7.17(\mathrm{~m}, 2 \mathrm{H}), 7.27-7.36(\mathrm{~m}, 6 \mathrm{H}), 7.55(\mathrm{dd}, J=0.6$, $7.8 \mathrm{~Hz}, 1 \mathrm{H}), 8.51$ (br, 1H). ${ }^{13} \mathrm{C}-\mathrm{NMR}, 50 \mathrm{MHz}: \delta 51.1,55.2,58.2,63.1,101.4,110.7,119.6,120.0$, $121.5,127.2,128.4(2 \mathrm{C}), 128.5,129.0,132.6,136.0,136.5,138.5]$.

To a solution of the alcohol (1.0 mmol, 1.0 equiv) and pyridine $\left(2.0 \mathrm{mmol}, 2.0\right.$ equiv) in dry $\mathrm{Et}_{2} \mathrm{O}$ $(10 \mathrm{~mL})$, methyl chloroformate $\left(1.5 \mathrm{mmol}, 1.5\right.$ equiv) dissolved in dry $\mathrm{Et}_{2} \mathrm{O}(1.0 \mathrm{~mL})$ was added dropwise at $0^{\circ} \mathrm{C}$. The reaction mixture was stirred for $24 \mathrm{~h}$ at $\mathrm{rt}$ then quenched with water $(5 \mathrm{~mL})$, extracted with AcOEt, dried over $\mathrm{Na}_{2} \mathrm{SO}_{4}$, concentrated under reduced pressure and purified by flash chromatography (silica gel, cycloxexane:AcOEt 9:1) to give $\mathbf{1 2}$ as a yellow oil in 65\% yield, $237 \mathrm{mg}$. 
${ }^{1} \mathrm{H}-\mathrm{NMR}\left(200 \mathrm{MHz}, \mathrm{CDCl}_{3}\right): \delta 3.09(\mathrm{~d}, J=5.8 \mathrm{~Hz}, 2 \mathrm{H}), 3.59(\mathrm{~s}, 2 \mathrm{H}), 3.70(\mathrm{~s}, 2 \mathrm{H}), 3.75(\mathrm{~s}, 3 \mathrm{H})$, $4.58(\mathrm{~d}, J=5.6 \mathrm{~Hz}, 2 \mathrm{H}), 5.65-5.85(\mathrm{~m}, 2 \mathrm{H}), 6.34(\mathrm{~s}, 1 \mathrm{H}), 7.08-7.22(\mathrm{~m}, 2 \mathrm{H}), 7.28-7.32(\mathrm{~m}, 6 \mathrm{H})$,

$7.50(\mathrm{~d}, J=7.2 \mathrm{~Hz}, 1 \mathrm{H}), 8.39$ (br, $1 \mathrm{H}) .{ }^{13} \mathrm{C}-\mathrm{NMR}\left(75 \mathrm{MHz}, \mathrm{CDCl}_{3}\right): \delta 50.8,54.8,57.9,67.8,101.4$, 110.6, 119.5, 120.0, 121.4, 126.7, 127.1, 128.3, 128.4, 128.9, 132.9, 135.9, 136.5, 138.5, 155.6. IR (neat): $v$ 3407, 3058, 3027, 2952, 2813, 1747, 1455, 1267, 1137, $943 \mathrm{~cm}^{-1}$. Anal. calcd for $\left(\mathrm{C}_{22} \mathrm{H}_{24} \mathrm{~N}_{2} \mathrm{O}_{3}\right)$ : C, 72.50; H, 6.64; N, 7.69; Found: C, 72.48; H, 6.59; N, 7.63.

\section{Preparation of 2-benzyl-4-vinyl-2,3,4,9-tetrahydro-1H- $\beta$-carboline (14)}

The reaction was carried out at $\mathrm{rt}$ under nitrogen atmosphere. A solution of $[\mathrm{PdCl}(\pi \text {-allyl })]_{2}\left(5 \cdot 10^{-3}\right.$ mmol, 0.05 equiv) and triphenyl phosphine $\left(2.2 \cdot 10^{-2} \mathrm{mmol}, 0.22\right.$ equiv) in anhydrous $\mathrm{CH}_{2} \mathrm{Cl}_{2}(1.0$ $\mathrm{mL}$ ) was stirred for $30 \mathrm{~min}$ and then 12 (0.1 mmol, 1.0 equiv), $\mathrm{Li}_{2} \mathrm{CO}_{3}(0.2 \mathrm{mmol}, 2.0$ equiv) and BSA (0.2 mmol, 2.0 equiv) were added. The resulting reaction mixture slowly turned form yellow to orange. The reaction was stirred for $4 \mathrm{~h}$ at $\mathrm{rt}$ (complete consumption of $\mathbf{1 2}$ as judged by TLC) and then quenched with water $(4 \mathrm{~mL})$ and extracted with AcOEt. The combined organics were dried over $\mathrm{Na}_{2} \mathrm{SO}_{4}$ and concentrated under reduced pressure. The crude was purified by passage through a pad of silica gel (silica gel, $n$-Hexane:AcOEt 90:10) affording 14/15 as a orange oil in 91\% yield, $26 \mathrm{mg}\left(\mathbf{1 4} / 15\right.$ ratio $>50 / 1$, determined by ${ }^{1} \mathrm{H}-\mathrm{NMR}$ on the crude mixture).

${ }^{1} \mathrm{H}-\mathrm{NMR}\left(200 \mathrm{MHz}, \mathrm{CDCl}_{3}\right): \delta 2.58(\mathrm{dd}, 1 \mathrm{H}, J=11.6,7.0 \mathrm{~Hz}), 2.89(\mathrm{dd}, 1 \mathrm{H}, J=11.6,4.8 \mathrm{~Hz})$, 5.34-5.55 (m, 2H), $3.68(\mathrm{~d}, J=3.0 \mathrm{~Hz}, 3 \mathrm{H}), 5.03-5.25(\mathrm{~m}, 2 \mathrm{H}), 5.88(\mathrm{ddd}, J=10.0,8.0,1.8 \mathrm{~Hz}$, 1H), 6.90-7.12 (m, 2H), 7.15-38 (m, 6H), $7.45(\mathrm{~d}, J=8.2 \mathrm{~Hz}, 1 \mathrm{H}), 7.60$ (br s, 1H). ${ }^{13} \mathrm{C}-\mathrm{NMR}(50$ $\left.\mathrm{MHz}, \mathrm{CDCl}_{3}\right): \delta 38.5,50.1,57.3,61.9,110.0,110.7,115.3,119.1,119.3,121.2,127.1,127.2$, $128.3,129.0,132.1,136.0,138.4,140.4$.

IR (neat): v 3403, 3060, 2932, 2802, 1637, 1453, 1330, 1228, 1012, $915 \mathrm{~cm}^{-1}$. LC-ESI-MS: 289 $(\mathrm{M}+1)$. Anal. calcd for $\left(\mathrm{C}_{20} \mathrm{H}_{20} \mathrm{~N}_{2}\right)$ : C, 83.30; H, 6.99; N, 9.71; Found: C, 83.25; H, 6.94; N, 9.68.

\section{Preparation of 2-benzyl-4-vinyl-1,2,3,4-tetrahydro-pyrazino[1,2-a]indole (15)}

The reaction was carried out at $\mathrm{rt}$ under nitrogen atmosphere. A solution of $[\mathrm{PdCl}(\pi \text {-allyl })]_{2}\left(5 \cdot 10^{-3}\right.$ mmol, 0.05 equiv) and triphenyl phosphine $\left(2.2 \cdot 10^{-2} \mathrm{mmol}, 0.22\right.$ equiv) in anhydrous DMF (3.0 $\mathrm{mL}$ ) was stirred for $30 \mathrm{~min}$ and then 12 ( $0.1 \mathrm{mmol}, 1.0$ equiv) and $\mathrm{Cs}_{2} \mathrm{CO}_{3}$ (0.2 mmol, 2.0 equiv) were added. The resulting reaction mixture slowly turned form yellow to orange. The reaction was stirred for $3 \mathrm{~h}$ at $50^{\circ} \mathrm{C}$ (complete consumption of $\mathbf{1 2}$ as judged by TLC) and then quenched with water $(4 \mathrm{~mL})$ and extracted with AcOEt. The combined organics were dried over $\mathrm{Na}_{2} \mathrm{SO}_{4}$ and 
concentrated under reduced pressure. The crude was purified by passage through a pad of silica gel (silica gel,, $n$-Hexane:AcOEt 99:1) affording 14/15 as a yellow oil in 85\% yield, $24 \mathrm{mg}$ (14/15 ratio $=1 / 8$, determined by ${ }^{1} \mathrm{H}-\mathrm{NMR}$ on the crude mixture).

${ }^{1} \mathrm{H}-\mathrm{NMR}\left(200 \mathrm{MHz}, \mathrm{CDCl}_{3}\right): \delta 2.86(\mathrm{t}, 2 \mathrm{H}, J=3.2 \mathrm{~Hz}), 3.54-3.66(\mathrm{~m}, 3 \mathrm{H}), 3.90(\mathrm{~d}, 1 \mathrm{H}, J=14.4$ $\mathrm{Hz}), 4.70-4.77(\mathrm{~m}, 1 \mathrm{H}), 5.10(\mathrm{~d}, 1 \mathrm{H}, J=10.6 \mathrm{~Hz}), 5.16(\mathrm{~d}, 1 \mathrm{H}, J=2.8 \mathrm{~Hz}), 5.96(\mathrm{ddd}, 1 \mathrm{H}, J=10.6$, 8.2, $2.8 \mathrm{~Hz}), 6.11(\mathrm{~s}, 1 \mathrm{H}), 6.95-7.01(\mathrm{~m}, 2 \mathrm{H}), 7.21-7.29(\mathrm{~m}, 6 \mathrm{H}), 7.43-7.48(\mathrm{~m}, 1 \mathrm{H}) .{ }^{13} \mathrm{C}-\mathrm{NMR}(50$ $\left.\mathrm{MHz}_{\mathrm{CDCl}}\right): \delta$ 51.0, 55.4, 56.0, 61.4, 96.1, 109.9, 116.3, 119.1, 119.3, 119.7, 126.7, 127.8, 127.9, 128.2, 133.7, 135.2, 136.9, 137.0. IR (neat): v 3078, 3058, 2800, 2746, 1642, 1456, 1331, 1014, $923 \mathrm{~cm}^{-1}$. LC-ESI-MS: $289(\mathrm{M}+1)$. Anal. calcd for $\left(\mathrm{C}_{20} \mathrm{H}_{20} \mathrm{~N}_{2}\right): \mathrm{C}, 83.30 ; \mathrm{H}, 6.99 ; \mathrm{N}, 9.71$; Found: C, 83.31; H, 6.98; N, 9.70.

1. Trost, B. M.; Fraisse, P. L.; Ball, Z. T. Angew. Chem. Int. Ed. 2002, 41, 1059-1061.

2. Malkow, A. V.; Davis, S. L. ; Baxendale, I., R.; Mitchell, W. L.; Kočovský, P. J. Org. Chem. 1999, 64, 2751-2764.

3. Suzuki, K.; Unemoto, M.; Hagiwara, M.; Ohyama, T.; Yokoyama, Y.; Murakami, Y. J. Chem. Soc. Perkin Trans. 1 1999, 1717-1724.

4. Shimoji, Y.; Hashimoto, T.; Furukawa, Y.; Yanagisawa, H. Heterocycles 1993, 36, 123-132. 